\title{
Audit to look at the effectiveness of enteral feeding in allogenic bone marrow transplant patients admitted to the bone marrow transplant unit at St. James Hospital in Leeds
}

\author{
N. Scott \\ Nutrition and Dietetic Department, St. James University Hospital, Beckett Street, Leeds LS9 7TF, UK
}

Currently there are many controversies surrounding nutritional support in bone marrow transplant patients. Historically parenteral nutrition (TPN) has been the favoured route in this patient group, although enteral nutrition is being now being considered more often due to the lower associated risk of infection, especially in these patients who are immuno-compromised ${ }^{(1)}$. In view of this move towards enteral feeding, our current practice on the bone marrow transplant unit is to use the enteral route, by placing a naso-gastric (NG) feeding tube (NGT).

The information was gathered retrospectively over a 1-year period from April 2005 to April 2006 for those patients who were referred to the dietetic department according to our referral criteria. Information gathered included sex, age, type of transplant, weight on admission, weight on discharge, route of nutritional support, day post-transplant nutritional support was initiated, length of time patient received artificial nutritional support for and the percentage of calories and protein from estimated requirements oral intake provided when artificial nutritional support was stopped.

Over this year eighteen out of twenty-one patients were referred for nutritional support. The remaining three were not referred by nursing staff as it was felt they were eating adequately.

NG feeding was recommended in $100 \%$ of the patient group, with NGT only successfully placed in $67 \%$ (twelve patients). Of the eighteen patients, two refused to have an NGT passed and the other four could not tolerate the procedure due to the severity of mucositis. Of the remaining six patients, three received oral support and three received TPN. The mean day that artificial nutrition was initiated on was day +2 post-transplant (range 0-13) and the overall mean length of time for artificial feeding was $10 \mathrm{~d}$. For those who were NG-fed mean time on feed was $10 \mathrm{~d}$ whereas for those who received TPN mean time of feeding was $11.5 \mathrm{~d}$. Only $13 \%$ of the patients who received artificial nutrition had their feeding stopped appropriately with an adequate oral intake of over $50 \%$ of estimated requirements. Of the patients who had NG tubes placed, they had the least weight loss overall with a mean loss of $2.36 \mathrm{~kg}$. Those who received TPN or oral support lost a mean of $7.9 \mathrm{~kg}$ in total.

In summary this audit shows that NG feeding can be an effective way of providing artificial nutrition to this group of patients. It shows that NG feeding from day 0 onwards can be achieved and an earlier placement pre-mucositis developing may improve patient's toleration to the procedure. Sefcick et al. ${ }^{(2)}$ also recommend the placement of feeding tubes by day +1 in this patient group. By passing NGT earlier and replacing those tubes which were displaced prior to achieving adequate oral intake then weight loss could be limited further.

1. Muscaritoli M, Grieco G, Capria S, Iori AP \& Fanelli FR (2002) Am J Clin Nutr; 75, No 2: $183-190$

2. Sefcick A, Anderson D, Byrne JL, Teahon K \& Russell NH (2001) Bone Marrow Transplant 28, 1135-1139. 